\title{
REVISIONES
}

\section{Aproximación al estudio del desarrollo educativo desde los Ayuntamientos y en el contexto español}

\author{
Approximation to the study of educational development \\ from the city councils and in the Spanish context
Aproximação ao estudo do desenvolvimento educacional desde os Conselhos e no contexto espanhol

\author{
José Luís Muñoz Moreno
}

\begin{abstract}
Departamento de Didáctica y Organización Escolar, Facultad de Filosofía y Ciencias de la Educación, Universidad de Valencia (España),+34 963983712, jose.1.munoz@uv.es
\end{abstract}

\begin{abstract}
RESUMEN
El artículo presenta el principal resultado de un estudio realizado, cuyo propósito ha sido indagar en el desarrollo educativo de los municipios a través de la actuación implementada por los Ayuntamientos en el contexto español. Contiene la concreción y validación de una propuesta de Modelo de estadios de desarrollo educativo, que ha supuesto la revisión bibliográfica, 17 entrevistas en profundidad, 2 grupos de discusión, la aplicación piloto del Modelo en 3 Ayuntamientos y su validación en la práctica. Los resultados remarcan que son requisitos clave del desarrollo educativo el disponer de planteamientos institucionales contextualizados, la flexibilidad de las estructuras organizativas y un sistema relacional abierto a la innovación.
\end{abstract}

Palabras clave: desarrollo educativo, ayuntamiento, municipio, aprendizaje, estadios.

\section{ABSTRACT}

The article presents the main results of a study whose purpose has been to investigate the educational development of the municipalities through the actions implemented by the City Councils in the Spanish context. It contains the implementation and validation of a proposed model of stages for educational development, which has resulted in the literature review, 17 in-depth interviews, 2 discussion groups, the pilot implementation of the Model in 3 municipalities, and its validation in practice. Open-ended results indicate some key requirements for educational development: contextualized institutional approaches, flexibility of organizational structures, and a relational system open to innovation.

Keywords: educational development, city council, municipality, learning, stages.

\section{RESUMO}

Apresenta-se o principal resultado do estudo que teve como objetivo investigar o desenvolvimento educativo dos municípios por meio da atuação implementada pelos Conselhos no contexto espanhol. Contém a implementação e validação de uma proposta de Modelo de ciclos de desenvolvimento educacional, na medida em que envolveu uma revisão bibliográfica, 17 entrevistas em profundidade, 2 grupos de discussão, a aplicação piloto do Modelo em 3 Conselhos e sua validação na prática. Resultados realçam que o dispor de abordagens institucionais contextualizadas, a flexibilidade das estruturas organizacionais e um sistema relacional aberto à inovação são requisitos-chave do desenvolvimento educativo.

Palavras-chave: desenvolvimento educacional, conselhos, município, aprendizagem, ciclos. 


\section{INTRODUCCIÓN}

España tiene un total de 8.116 municipios, de los que 29 tienen más de 200.000 habitantes, 7.717 menos de 20.000 y 370 entre 20.000 y 200.000 (INE, 2012). En los últimos años, sus municipios han sufrido desajustes entre las funciones asumidas por los Ayuntamientos y las competencias que legalmente les correspondían, problemas derivados de la falta de financiación y limitaciones en relación a las propias capacidades de autoorganización (FEMP, 2006 y 2011). En el ámbito de la educación, aún a pesar de existir entornos socioeducativos con características similares, la gestión local es muy diversa y distinta. El compromiso municipal con la educación, la participación educativa promovida, las actuaciones dinamizadas o las preocupaciones por la mejora, entre otras cuestiones, representan aspectos que distinguen a unos municipios de otros y evidencian las diferentes maneras de gestionar la educación desde los Ayuntamientos.

La prestación del servicio educativo habitualmente se desarrolla desde las prescripciones y orientaciones realizadas por el Estado y las Comunidades Autónomas; pero cada vez son más los Ayuntamientos que se muestran dispuestos a desempeñar un papel de primer orden en las políticas educativas (Albaigés, 2013). Toma fuerza así la idea del municipio como agente educativo, fuente de aprendizaje y también de cohesión y convivencia (Gairín, 2005), al representar un espacio articulador de la diversidad y posibilitador de la participación ciudadana (Lavín y Nájera, 2003) gracias tanto a su proximidad como a la implementación de políticas y economías educativas, el diseño y desarrollo de planes y programas educativos y la evaluación de resultados (Carnicero, 2001), superando incluso el marco competencial municipal, asociado habitualmente con: la conservación, el mantenimiento y la vigilancia de los edificios escolares; la colaboración con los centros educativos en el impulso de actividades extraescolares; la promoción de la relación entre la programación de los centros y el entorno; el posibilitar el uso de los centros fuera del horario escolar; el garantizar la orientación psicopedagógica y profesional del alumnado; la cooperación con los gobiernos autonómicos en la obtención de solares para construir nuevos centros y establecer convenios con otras administraciones sobre enseñanzas de régimen especial; la creación y desarrollo de escuelas de educación infantil; la representación del Ayuntamiento en los Consejos Escolares de los centros y la potenciación de la participación ciudadana en el Consejo Escolar municipal.

La inquietud sobre la educación como objeto de atención de los Ayuntamientos se despierta, pero también lo hace la reflexión en relación a su sentido, posibilidades y límites, situada en el enfoque de la participación social en educación (Muñoz, 2012; Apple y Beane, 1997; San Fabián, 1994). Conviene, de acuerdo con Subirats (2007), construir un municipio para todos, donde todos puedan lograr su plenitud como personas siendo parte de una comunidad plural de individuos autónomos. En opinión de Carneiro (1999), hay que fortalecer el municipio como instancia de conocimiento y creatividad en el arte de vivir juntos, resurgir a municipios educadores que acogen y vencen la exclusión, y redescubrir la democracia urbana compaginando progreso, participación, crecimiento, calidad, libertad, responsabilidad, gobernabilidad y mediatización en la vida pública.

Los Ayuntamientos, constantemente enfrentados a situaciones educativas nuevas, cambiantes y complejas, como consecuencia de una realidad dinámica y una sociedad exigente, han de impulsar las transformaciones requeridas asegurando propuestas educativas de calidad e interés ciudadano, coordinadas y focalizadas, especialmente, en los colectivos 
más vulnerables, optimizando recursos, y generando y gestionando conocimiento y aprendizaje de forma colaborativa (Bossi, Fuertes y Serrano, 2001; Mackay y Hows, 2005). La responsabilidad de los Ayuntamientos se dirige así al conjunto de la ciudadanía, no sólo de la población escolarizada obligatoriamente, y abarca los ámbitos formal, no formal e informal de la educación. Por eso, la gestión de la educación debe combinar nuevas fórmulas de participación con un importante protagonismo de la ciudadanía, sea desde la participación mixta o bien desde la base exclusivamente personal (Tonucci, 1998; Kahn, 1999; Dienel y Harms, 2000; Soler, 2006), pues se trata de progresar en nuevos modelos de gestión en la línea de una administración de calidad y más focalizada en el ciudadano.

Los Ayuntamientos, en tanto que gestores de las políticas públicas de educación en el contexto de los municipios, y en caso de tener realmente preocupación por la mejora educativa, han de ser mediadores formativos y culturales, enraizándose en la comunidad, abriéndose a las funciones que la sociedad les demanda (Carvajal, 2011; Comellas, 2010) y respondiendo operativamente a los problemas educativos. Más aún, cuando la escolaridad transciende las etapas obligatorias, crece la oferta y demanda formativas y se reivindica una mejor calidad en los procesos y servicios educativos. La educación desde los Ayuntamientos y en los municipios supone un desafío que debe reinventarse nuevamente (Caballo, 1999), educando para la vida desde la propia vida, impulsando el aprendizaje a lo largo de toda la vida y para todos, relacionándose con la inserción y promoción laborales, incidiendo en la participación democrática y social, y comprometiéndose con la equidad y la igualdad de oportunidades.

\section{DESCENTRALIZACIÓN Y AUTONOMÍA INSTITUCIONAL PARA EL DESARROLLO EDUCATIVO}

La descentralización de la educación se sostiene en la necesidad de contextualizar los contenidos curriculares, pero también en la flexibilización de unos servicios educativos que operan en entornos constantemente cambiantes, en la participación de la comunidad y la integración de recursos. Refuerza la democracia por aproximar los asuntos públicos a instituciones cercanas y mejora la eficiencia al descongestionar los poderes centrales. Bonal y Verger (2014) entienden que la no aproximación de la gestión educativa a los Ayuntamientos hace difícil establecer mecanismos de consenso en favor de la eficacia y la equidad. La descentralización nos sitúa, de acuerdo con Gimeno (2005), en la perspectiva de articular proyectos propios y de reclamar poder para hacerlos posibles desde el derecho a las diferencias culturales.

Ya no es suficiente satisfacer las necesidades básicas, sino que además hay que responder a los retos planteados y al desafío de compatibilizar el que seamos diferentes siendo iguales para poder ser igualmente diferentes (Gimeno, 2005). Se precisa de nuevos marcos institucionales que reconozcan el protagonismo de los Ayuntamientos en definir, planificar y gestionar la política educativa de un país. Los procesos de descentralización y autonomía institucional entran en esta lógica, que es también la lógica de la corresponsabilidad con la educación.

Países como Finlandia, Islandia, Dinamarca, Noruega, Suecia, Polonia, Hungría, Reino Unido o Corea reconocen la importancia de la participación de los Ayuntamientos en la educación (Comas, Abellán y Plandiura, 2014; Gripenberg y Lizarte, 2012; Pedró, 2008). 
Algunos de los buenos resultados que estos países tienen en las pruebas internacionales PISA también se explica por la autonomía concedida a los municipios y los centros escolares para desarrollar contenidos, y en la asignación de recursos (Schleicher, 2005). En cualquier caso, la descentralización debiera garantizar el compromiso de una educación para todos, equidad, igualdad de oportunidades, cohesión social y calidad; pero también que las competencias descentralizadas puedan ser asumidas con la máxima garantía y responsabilidad de parte de los órganos de gobierno y gestión correspondientes.

En España el ordenamiento jurídico en materia educativa concede un protagonismo relevante al Estado en el período preconstitucional, y a las Comunidades Autónomas posteriormente; lo que dificulta, de esta forma, la descentralización hacia los municipios de la mano de los Ayuntamientos (Velázquez, 2008; Subirats, 2001). De hecho, las recientes Ley de Racionalización y Sostenibilidad de la Administración Local y Ley Orgánica para la Mejora de la Calidad Educativa, ambas del año 2013, continúan sin considerar a los Ayuntamientos como Administración educativa y reducen la intensidad y el protagonismo de los municipios ante la política educativa.

La gestión de la educación desde la participación de los Ayuntamientos, total o parcialmente, contribuye a la mejora personal y a la evolución social, con el horizonte de buscar la felicidad de las personas en su entorno y una sociedad más justa y cohesionada. La diversidad de las realidades educativas invita a pensar no sólo en soluciones uniformes, aún a pesar de que la implicación educativa de los Ayuntamientos no depende únicamente de la mayor o menor centralización o descentralización del sistema educativo. La honestidad y la sinceridad de los protagonistas, la capacidad para mantener colaboraciones y establecer sinergias, la búsqueda de consensos entre quienes deciden o la implicación de las comunidades educativas, son otros factores influyentes.

La mayor autonomía institucional de los Ayuntamientos ante la educación queda justificada por razones de tipo filosófico - al priorizar la opción de autonomía y descentralización otorgada a las dimensiones personales-, social -al profundizar en la democracia y llevando la toma de decisiones públicas al ciudadano-, político -al transferir los recursos y los procesos de gestión-, psicopedagógico -al motivar a las personas para que participen en construir sus realidades-, y económico -al rendir cuentas, delimitar responsabilidades institucionales e innovar (Batanaz, 2006). La autonomía institucional se apoya, así, en la democratización de la sociedad, el respeto a las particularidades socioculturales más próximas y el principio de equidad. De acuerdo con Batanaz (2006), una autonomía consistente ha de poder relacionarse con los principios y prácticas de la acción educativa que se lleva acabo, ejercerse a partir de metas coherentes con los propósitos sociales del sistema educativo que sean asumidas por todos y elaborarse con propuestas propias de acción y realistas al tenor de las posibilidades y los recursos disponibles.

Descentralización y autonomía institucional se muestran imprescindibles para que los Ayuntamientos puedan actuar en favor del desarrollo educativo que, tomando de partida los trabajos de De la Riva (2004), Nisbet (2008) y Heras y otros (2008), se considera como un proceso global, holístico y capaz de generar cambios para mejorar las condiciones educativas de los municipios y la ciudadanía. Por eso, coincidimos con Enríquez y Gallichio (2006), y Enríquez (2003), en entender que el desarrollo educativo es un proceso de concertación entre los diferentes agentes y actores socioeducativos que interactúan en el municipio para impulsar, desde la participación activa y responsable de todos, un proyecto educativo común que genera crecimiento, equidad, igualdad de 
oportunidades, cohesión, convivencia, calidad, sostenibilidad, conocimiento, aprendizaje y cambio; y cuyos objetivos primordiales tienen que ver con mejorar la calidad educativa de la ciudadanía y del municipio, así como afrontar oportunamente los retos educativos que, global y localmente, se plantean a estos.

Comprender el desarrollo educativo de esta forma supone adoptar una visión a medio y largo plazo que marque el horizonte educativo al que se aspira y sobre el que cabe avanzar de forma gradual; pero también, requiere de trabajo conjunto para construir unos municipios realmente educativos y educadores, democráticos y participativos, diversos e inclusivos. En el fondo, se trata de mejorar las condiciones educativas en todas las esferas y niveles, garantizando la educación como derecho para todos, tal y como sostiene la Declaración Universal de los Derechos Humanos (1948). Todavía más, si cabe, cuando la inversión en educación, con la OEI (2005) y Filmus y Serrani (2009), ayuda a crear empleo y crecimiento económico, facilita mejorar los indicadores de salud, por incluir a los colectivos más vulnerables, y reducir la discriminación y la exclusión, sea del tipo que sea, y refuerza una base más sólida para la democracia.

\section{DISEÑO Y DESARROLLO DEL ESTUDIO}

La investigación realizada ha estudiado el desarrollo educativo de los municipios desde la actuación implementada por los Ayuntamientos. En concreto, sus objetivos han sido: analizar los principales elementos que se vinculan a la mejora de la gestión educativa de los municipios, y diseñar y validar un Modelo de estadios de desarrollo educativo.

La perspectiva metodológica seguida se aproxima a planteamientos de carácter cualitativo y descriptivo, donde el trabajo de campo ha tenido importantes coincidencias con los estudios "survey" y la óptica de investigación focalizada en la mejora de la política y la práctica educativas ("policy - oriented research").

El proceso de investigación ha supuesto la revisión bibliográfica para identificar los fundamentos teóricos de referencia, así como la implementación de un trabajo de campo orientado al diseño y la validación de la propuesta de Modelo de estadios de desarrollo educativo mediante entrevistas en profundidad, grupos de discusión, la aplicación piloto en Ayuntamientos y su validación en la práctica.

La instrumentalización ha contado con un guión de entrevista (estructurada, informativa y de investigación) que se ha interesado por cuestiones asociadas al desarrollo educativo (algunas de las cuales quedan recogidas en el Cuadro 1); un guión de discusión con cuestiones sobre la opinión general, adecuación, terminología, complejidad, claridad y comprensión de la propuesta de Modelo de estadios; un instrumento de autoevaluación para aplicar el Modelo a modo de pilotaje en Ayuntamientos y que pregunta por 26 cuestiones distintas (Cuadro 1); y un Guión de validación con preguntas abiertas sobre la utilidad del Modelo propuesto en la práctica (univocidad, importancia y distancia).

El muestreo tomado en consideración ha sido no probabilístico, bajo una modalidad intencional que limita la generalización de la muestra a la respectiva representatividad de los informantes participantes. En las entrevistas han participado 17 informantes seleccionados bajo un múltiple criterio (pertenencia a municipios de tamaño pequeño, mediano y grande, formar parte del movimiento de ciudades educadoras, diversidad de cargos políticos y de gestión ocupados, así como de instituciones representadas: Asociación Internacional 
Estudios Pedagógicos XLII, $\mathrm{N}^{\circ}$ 1: 355-366, 2016

APROXIMACIÓN AL ESTUDIO DEL DESARROLLO EDUCATIVO DESDE LOS AYUNTAMIENTOS Y EN EL CONTEXTO ESPAÑOL

Cuadro 1. Cuestiones para el análisis del desarrollo educativo.

\begin{tabular}{|l|l|}
\hline Valoración del desarrollo educativo & Importancia de la educación \\
\hline Implicación en educación & Trabajo por la mejora educativa \\
\hline Dificultades para participar en educación & Actuaciones educativas realizadas \\
\hline Procesos de aprendizaje promovidos & Implicación en aspectos curriculares \\
\hline Difusión de la actuación & Dificultades de acceso a la información \\
\hline Programa educativo municipal & Resultados de la actuación \\
\hline Inversión en educación & Planteamientos institucionales \\
\hline Recursos humanos dedicados & Protagonistas del sistema relacional \\
\hline Agente de cambio en educación & Procesos de participación \\
\hline Comunicación & Función organizativa: planificación \\
\hline Función organizativa: toma de decisiones & Función organizativa: evaluación \\
\hline Estructuras organizativas & Cultura \\
\hline Estrategias para la mejora & Otros comentarios y observaciones \\
\hline
\end{tabular}

de Ciudades Educadoras, Ministerio de Educación español, Consejo Escolar del Estado, Federación Española de Municipios y Provincias, Federación Española de Universidades Populares, Ayuntamientos y Universidades), y su triangulación en los niveles político, técnico municipal de educación y miembro de la comunidad educativa. En los 2 grupos de discusión han participado 11 informantes pertenecientes a municipios de tamaño distinto, con experiencia en la temática de análisis, trayectorias profesionales relevantes y capaces de aportar orientaciones prácticas (5) y teóricas (6) de interés. En la aplicación piloto del Modelo en Ayuntamientos participaron 3 municipios de tamaño diverso, con unos Servicios Municipales de Educación muy implicados y experiencias educativas relevantes (Proyecto Educativo de Ciudad, Pacto Local por la Educación, Plan Educativo de Entorno, Consejo Educativo, etc.). Y en la validación del Modelo, los informantes fueron 12 técnicos municipales de educación con un alto nivel de calificación y capaces de aportar información significativa, reflexiones de interés, validez contextual y variedad de perspectivas.

La información recabada ha sido tratada desde un modelo de contrastación a partir de identificar categorías de análisis (coincidentes con las cuestiones señaladas en el anterior Cuadro 1) y considerar las ideas relevantes, profundizando en divergencias, coincidencias, valoraciones y propuestas de los informantes participantes. Este mismo criterio se ha seguido también en la aplicación piloto del Modelo en Ayuntamientos y en su validación por parte de técnicos municipales de educación.

\section{RESULTADOS}

La triangulación de los resultados obtenidos mediante el trabajo de campo desemboca en la propuesta de Modelo de estadios de desarrollo educativo que concretamos aquí (Figura 1), y que refleja cómo la implicación de los Ayuntamientos en la educación puede transitar 
entre distintos estadios. El Modelo se focaliza en la participación y el compromiso de los Ayuntamientos con la educación, por entenderse fundamentales para el logro de la mejora; también considera que la autonomía ejercida y la voluntad política y comunitaria son mayores cuando se avanza hacia estadios superiores. Procura ser una herramienta de ayuda y utilidad al servicio de responsables políticos y técnicos de educación en los municipios y algunas de las tendencias que se señalan a continuación son las que caracterizan los estadios establecidos a partir del estudio realizado.

Figura 1. Modelo de estadios de desarrollo educativo (elaboración propia).

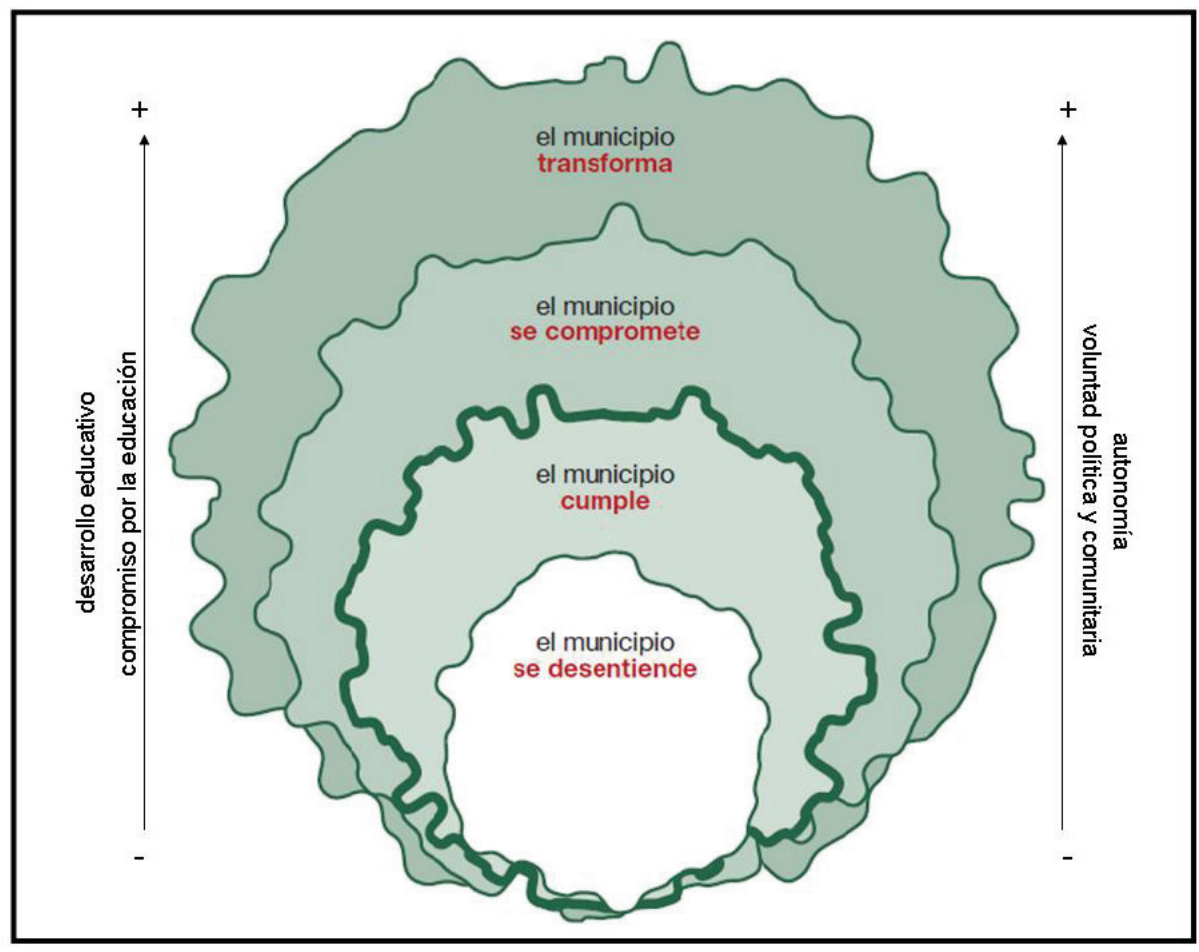

La política educativa, marcada por el Gobierno del Estado y las Comunidades Autónomas en España, define las competencias atribuidas a los Ayuntamientos en educación. Estos se muestran como entes dependientes de las decisiones educativas tomadas por parte de las administraciones superiores y su protagonismo se reduce al cumplimiento de la normativa y la legislación desde las competencias transferidas. Sin embargo, en el heterogéneo contexto municipal, puede pasar que existan Ayuntamientos que no cumplan adecuadamente con esas competencias: es cuando nos referimos a un estadio de desarrollo educativo en que "el municipio se desentiende" de la educación, de las obligaciones correspondientes y no se implica. En este estadio, que debiera ser de cumplimiento normativo, se encuentran quienes tienen un escaso interés por la educación 
y poca preocupación por la mejora educativa, denotando incluso incompetencia política y técnica, evidenciando que la educación no es una prioridad ni contribuye al progreso.

Superado este estadio previo, hay comportamientos de Ayuntamientos que tratan de aumentar su protagonismo e implicación con la educación. Por ejemplo, realizando actuaciones educativas más allá del marco escolar, dedicando esfuerzos a mejorar la calidad de sus programas educativos, desarrollando órganos de participación, dando soporte a las organizaciones socioeducativas, etcétera, lo que desprende un cierto interés por favorecer el desarrollo educativo. El primer estadio del Modelo, "el municipio cumple", queda identificado con los Ayuntamientos que desempeñan un papel secundario con respecto a la educación. No desentendiéndose de ella, se esfuerzan por cumplir oportunamente con la aplicación de las competencias que tienen asignadas. Aun así juegan un papel subsidiario y escasamente transcendente para el desarrollo educativo. La voluntad política y comunitaria, y la participación existente, están limitadas al cumplimiento de las obligaciones y los deberes legislativos. Los Ayuntamientos actúan como continentes de la acción educativa aunque no siempre con unas actitudes positivas, tal y como ocurre cuando no destinan recursos suficientes al cumplimiento competencial, no participan activamente en los Consejos Escolares o no hacen previsiones para reservar suelo dónde construir equipamientos docentes, por señalar algunas de las situaciones más habituales. Esta concepción es la que ha prevalecido y aún hoy está muy presente en muchos de los Ayuntamientos españoles.

El segundo estadio, "el municipio se compromete", requiere una posición más activa de los Ayuntamientos, comprometiéndose con la educación, ejerciendo su potestad reglamentaria y de auto-organización, gestionando sus propios intereses, ejecutando financieramente y revisando sus actos y acuerdos. Aquí hay Ayuntamientos con Proyectos Educativos propios, programas de actividades educativas complementarias al currículum escolar y actividades extraescolares, programas formativos dirigidos a colectivos diversos y para diferentes ámbitos, etc. También, pueden destinar recursos para prevenir el absentismo escolar, apoyar la inclusión, fomentar la continuidad del alumnado en estudios postobligatorios, dinamizar la participación de la comunidad educativa, etc. El compromiso mantenido explicita una importante toma de conciencia que se concreta en actuaciones superadoras del marco escolar, pues existe voluntad política y comunitaria por poner la educación entre las prioridades locales. Quienes se ubican en este estadio se abren a modificar las propias estructuras de organización y gestión cuando es preciso, cambian las actitudes y prácticas necesarias, operan colectivamente y diseñan e implementan planes, programas y proyectos educativos, profundizando en temas como la equidad, la igualdad de oportunidades, la cohesión social, la convivencia, la educación permanente, el aprendizaje a lo largo de toda la vida, entre otras propuestas. Se adaptan así a los cambios producidos en la sociedad y a las exigencias del entorno, estableciendo sinergias en beneficio del desarrollo educativo.

Pese a no tratarse de un proceso simple ni sencillo, es posible avanzar desde acciones diversas capaces de generar cambios gradualmente. Llevar a cabo iniciativas educativas, intercambiar experiencias, crear y gestionar conocimiento o procurar facilitadores, ayuda a cambiar el discurso y la práctica desde la reflexión y el análisis. Cuando se establecen mecanismos y procedimientos de evaluación institucional, con compromisos reales con el cambio y la mejora educativa, podemos pensar en situaciones de un tercer estadio, "el municipio transforma", en donde los Ayuntamientos además aprenden organizacionalmente, 
haciendo más efectiva su implicación y efectividad. Aquí ya es un hecho asumido la incorporación de programas educativos que tratan la diversidad, las tecnologías del aprendizaje y la comunicación, el conocimiento del medio, la alimentación saludable, la práctica educativa y cultural, etc. Se lleva hasta la máxima expresión el ejercicio de autonomía institucional de que disponen los Ayuntamientos, revitalizando las estructuras de participación educativa, colaborando con las familias para garantizar el éxito escolar, centrando esfuerzos en los colectivos más vulnerables y en riesgo de exclusión, estimulando la coordinación y el trabajo en red de las organizaciones socioeducativas, etc. Es más, se trabaja cooperativamente e impulsan autoevaluaciones institucionales, requisitos básicos para el desarrollo que se presupone debe potenciarse en este último estadio. La educación pasa a ser el pilar de la política educativa local y, por eso, los Ayuntamientos se dotan de estructuras y sistemas de funcionamiento flexibles que sólo son posibles cuando se piensa en un programa educativo abierto y como instrumento de cambio.

No obstante, no todo es previsible ni puede planificarse en la realidad municipal, pues acontecen situaciones rutinarias y comportamientos imprevistos que, en ocasiones, son complejos y tienen difícil solución al requerir de habilidades distintas a las tradicionalmente utilizadas. El principal reto para los Ayuntamientos reside, así, en tener la habilidad suficiente para superar esta complejidad mediante la autoevaluación institucional y el aprendizaje organizacional. Los Ayuntamientos, de algún modo, han de satisfacer los fines establecidos pero también ampliar sus funciones educativas y la corresponsabilidad educativa que tienen. Esta posición conduce a explorar nuevas estrategias educativas y actuaciones formativas para vencer las resistencias al cambio que se puedan presentar y valorar la transformación educativa del municipio desde la visión de una mayor y mejor calidad para sus contextos de referencia e intervención.

\section{DISCUSIÓN Y CONCLUSIONES}

La propuesta de Modelo elaborada puede orientar la actuación educativa de los Ayuntamientos, pese a que la realidad municipal está constantemente expuesta al cambio y la incertidumbre. Ha de servir para comprender la realidad, analizar las disfunciones, superar las dificultades y orientar la intervención desde la contextualización. El estudio realizado ratifica la utilidad de plantear el análisis de realidades complejas desde la consideración de estadios de desarrollo educativo.

La relación de los Ayuntamientos con la educación se puede describir mediante indicadores varios asociados con cada uno de los estadios. Las diversas situaciones posibles pueden categorizarse en un "continuum" dónde se determinan posiciones más o menos características. Ahora bien, situarse en un estadio concreto no supone un óptimo funcionamiento si la realidad existente no se mejora con respecto a situaciones anteriores; es más, puede ser una circunstancia puntual, a razón de los cambios que el dinamismo de la realidad impone. Por eso, el estadio más adecuado es aquel que resulta más útil al Ayuntamiento para mejorar, al tenor de la situación que tiene y de sus aspiraciones.

Dado que la realidad socioeducativa y la implicación de los Ayuntamientos en la educación resultan más complejas que los modelos que la intentan explicar, la identificación de estadios diferentes no supone, necesariamente, que haya un orden de prelación y competencia entre los mismos. Los estadios pueden ser complementarios puesto que cada 
uno asume una conceptualización más amplia y una mayor profundidad en el análisis y la práctica educativa. Son, en el fondo, distintos niveles de análisis que se distinguen por la perspectiva de la participación de los municipios en la educación (Muñoz, 2012).

De otra parte, puede darse el caso de situaciones en que haya presencia simultánea de varios estadios en función de determinados aspectos de análisis. La necesidad de que los Ayuntamientos progresen hacia estadios superiores, más comprometidos y transformadores, queda justificada por cuanto la propuesta puede favorecer el aprendizaje de todos, la participación y la cooperación, y promover una educación más justa y humana. También, por los efectos positivos del desarrollo educativo para el desarrollo social, democrático y económico (Filmus y Serrani, 2009; OEI, 2005). Se trata de tener la posibilidad de responder con garantías a los cambios, las necesidades y las exigencias que se plantean en la realidad educativa.

Los comportamientos abiertos a la reflexión, la investigación y el análisis representan especificaciones de actitudes de cambio y preocupación por la mejora que debe guiar la actuación de los Ayuntamientos. El desarrollo educativo contempla como requisitos clave la adopción de planteamientos institucionales contextualizados y compartidos, la flexibilidad de las estructuras de organización y gestión, y un sistema relacional abierto a la innovación. Por eso, el Modelo propuesto denota permeabilidad, flexibilidad y variabilidad, adaptándose así mejor a la variopinta realidad municipal.

Los Ayuntamientos que operan en beneficio de un mejor desarrollo educativo se muestran convencidos de que la educación es un instrumento útil para la transformación socioeducativa y, en este sentido, la sitúan entre las máximas prioridades de sus políticas sociales. Las estrategias de participación educativa, autoevaluación institucional y aprendizaje organizacional promovidas por los Ayuntamientos para estimular el desarrollo educativo son eficaces porque contribuyen a compartir objetivos, diseñar e implementar planes, programas y proyectos conjuntamente, y promover el diálogo y el consenso entre todos los agentes y actores. Su éxito reside en la contextualización, la priorización en la actuación, la capacidad de respuesta ante las necesidades y la implicación de la comunidad.

Los Ayuntamientos representan, por otro lado, unidades de actuación idóneas con unas fortalezas vinculadas al mejor conocimiento de la realidad y las necesidades educativas. El emergente poder local, la tendencia a gestionar los asuntos públicos desde la proximidad, el prestigio de los servicios educativos y la capacidad para promover el debate y la mejora, son otras oportunidades que tienen para comprometerse con el desarrollo educativo. Como vertebradores de la actividad socioeducativa de los municipios, debieran afrontar decididamente los retos que hoy tienen ante sí: la equidad, la igualdad de oportunidades, la inclusión, la corresponsabilidad educativa y el aprendizaje a lo largo de toda la vida, en favor de una mejor convivencia, cohesión, participación democrática y justicia social.

\section{REFERENCIAS BIBLIOGRÁFICAS}

Albaigés, B. (2013). Descentralització de la política educativa: consolidació, debilitats $i$ crisi. Barcelona: Diputació de Barcelona - Fundació Carles Pi i Sunyer - Fundació Jaume Bofill.

Apple, M. W. y Beane, J. A. (Coords.). (1997). Escuelas democráticas. Madrid: Morata.

Batanaz, L. (2006), "Desarrollo de la autonomía de los centros educativos y estímulo de su responsabilidad", en Revista Sociedad Española de Pedagogía. Extraído de: http://goo. 
gl/41yCD8 Fecha de consulta: 09-06-2014.

Bonal, X. y Verger, A. (2014). L'agenda de la política educativa a Catalunya: una anàlisi de les opcions de govern 2011-2013. Barcelona: Fundació Jaume Bofill.

Bossi, A.; Fuentes, Y.; Serrano, C. (2001). El capital intelectual en el sector público. Documento presentado en II Congreso de la Asociación Española de Contabilidad Directiva, León, 4-6 julio.

Caballo, $M^{\mathrm{a}}$. B. (1999). Los municipios ante la educación: perspectivas para una propuesta educativa enraizada en su entorno. Innovación Educativa, No 9, 243-253.

Carneiro, R. (1999). Educación para el siglo XXI. Hacia un nuevo contrato social de ciudad. Sabadell: Ajuntament de Sabadell.

Carnicero, P. (2001). Gobierno, participación y control de los centros educativos. Evaluación de los consejos escolares de centro. Barcelona: Universidad de Barcelona (Tesis Doctoral: documento policopiado).

Carvajal, A. (2011). Apuntes sobre desarrollo comunitario. Málaga: Eumed.net - Universidad de Málaga.

Comas, M.; Abellán, C.; Plandiura, R. (2014). Consells escolars i participació de les families a l'escola. Una lectura marcada per la LOMCE. Barcelona: Fundació Jaume Bofill.

Comellas, $\mathrm{M}^{\mathrm{a}}$. J. (2010). El treball en xarxa: un model de recerca i acció participativa per promoure la cooperació de les famílies. Educar, $\mathrm{N}^{\circ}$ 45, 117-129.

De la Riva, F. (2004). Nuevos actores para otro mundo local posible. Revista Documentación Social, $133,31-52$.

Derechos Humanos. (1948), Declaración Universal de los Derechos Humanos, Naciones Unidas. Extraído de: http://www.un.org Fecha de consulta: 01-06-2014.

Dienel, P. y Harms, H. (2000). Repensar la democracia. Barcelona: Ediciones del Serbal.

Enríquez, A. (2003). Desarrollo local: hacia una nueva forma de desarrollo nacional y centroamericano. Alternativas para el Desarrollo, 80. San Salvador: FUNDE.

Enríquez, A. y Gallichio, E. (2006). Gobernanza y desarrollo local. En Carrizo, L. y Gallichio, E. (Eds.). Desarrollo local y gobernanza. Enfoques transdisciplinarios. Investigación y políticas para el desarrollo en América Latina. Montevideo: Corporación Andina de Fomento - Unesco.

FEMP. (2011), 10 $0^{a}$ Asamblea general de la FEMP, Madrid; Federación Española de Municipios y Provincias. Extraído de: www.asamblea.femp.es Fecha de consulta: 20/04/2014.

FEMP. (2006). La situación de los Ayuntamientos en España, sus carencias económicas y problemas de gestión: diagnóstico y propuestas en una perspectiva europea. Madrid: BCL - Federación Española de Municipios y Provincias.

Filums, D. y Serrani, E. (2009). Development, education and financing. Analysis of debt waps for social investment as an extra-budgetary education financing insturment. Buenos Aires: Fundación SES - OEI.

Gairín, J. (Coord.). (2005). La descentralización educativa: ¿una solución o un problema?. Madrid: Praxis.

Gimeno, J. (2005). ¿Qué se puede ganar descentralizando la educación?. ¿Es posible diferenciarse sin desigualdad?. En Gairín, J. (Coord.). La descentralización educativa. ¿Una solución o un problema? Madrid: Wolters Kluwer.

Gripenberg, M. y Lizarte, E. J. (2012). El sistema educativo de Finlandia y su éxito en la prueba PISA. Journal for Educators, Teachers and Trainers, Vol. 3, 14-24.

Heras, P. (Coord.); Fernández, E.; Costa, S.; Gil, E.; Fernández, D.; Herreros, T. (2008). La acción política desde la comunidad. Barcelona: Graó.

INE. (2012), Instituto nacional de estadística, Madrid; Ministerio de Economía y Competitividad. Extraído de: www.ine.es Fecha de consulta: 21-12-2012.

Kahn, U. (Ed.). (1999). Beyond the ballot box. London: Routledge.

Lavín, S. y Nájera, E. (2003). Educación, participación social y conocimiento: una aproximación 
Estudios Pedagógicos XLII, Nº 1: 355-366, 2016

APROXIMACIÓN AL ESTUDIO DEL DESARROLLO EDUCATIVO DESDE LOS AYUNTAMIENTOS Y EN EL CONTEXTO ESPAÑOL

desde los sujetos. Revista Latinoamericana de Estudios Educativos, № 1, 9-98.

Mackay, G. y Howes, T. (2005). Channeling work streams in the organization. Knowledge Management Review, Vol. 7, Issue 6, 20-23.

Muñoz, J. L. (2012). Ayuntamientos y desarrollo educativo. Madrid: Editorial Popular.

Nisbet, R. (2008). La formación del pensamiento sociológico. Buenos Aires: Amorrortu Eds.

OEI. (2005). Deuda externa por educación. Madrid: OEI - Fundación Sin Fronteras.

Pedró, F. (2008). Descentralització i municipalització de l'educació als països de l'OCDE. Barcelona: Diputació de Barcelona.

San Fabián, J. L. (1994). Participar más y mejor. Cuadernos de Pedagogía, № 224, 70-71.

Schleicher, A. (2005). La mejora de la calidad y de la equidad en la educación: retos y respuestas públicas. Madrid: Fundación Santillana.

Soler, A. (2006). La escuela, núcleo de la red educativa territorial. Aula de Innovación Educativa, $\mathrm{N}^{\circ}$ $152,29-31$.

Subirats, J. (2001). Educació i govern local. La importancia del territorio i de la comunitat en el paper de l'escola. Barcelona: CEAC.

Subirats, J. (2007). ¿Una ciudad educadora?. El País, jueves 17 de mayo, Edición Cataluña. Extraído de: http://goo.gl/SdcWt3 Fecha de consulta: 03-05-2014.

Tonucci, F. (1998). La ciudad de los niños. Madrid: Fundación Germán Sánchez.

Velázquez, J. Ma. (Dtor.). (2008). Guía del concejal de educación, Manual de consulta para gobernantes y técnicos. Madrid: MEC-FEMP. 